\title{
Manual responses and saccades in chronic and recovered hemispatial neglect: a study using visual search
}

\author{
Monika Harvey $^{\mathrm{a}, *}$, Bettina Olk ${ }^{\mathrm{b}}$, Keith Muir ${ }^{\mathrm{c}}$, Iain D. Gilchrist ${ }^{\mathrm{d}}$ \\ a Department of Psychology, University of Glasgow, Glasgow G12 8QB, UK \\ ${ }^{\mathrm{b}}$ Department of Psychology, University of British Columbia, Vancouver, BC, Canada \\ ${ }^{\mathrm{c}}$ Department of Neurology, Southern General Hospital, Glasgow, UK \\ ${ }^{\mathrm{d}}$ Department of Experimental Psychology, University of Bristol, Bristol, UK
}

Received 19 March 2001; accepted 5 September 2001

\begin{abstract}
Hemispatial neglect affects both the ability to respond to targets on the contralesional side of space and to programme saccades to such targets. In the current study, we looked in detail at saccade programming and manual reaction times (RTs) in a range of visual search tasks, in which task difficulty was systematically increased by changing the nature of the distractors. In condition 1, the target was presented with no distractors. In the other conditions, displays contained three distractors that were changed across conditions to manipulate similarity to the target and so task difficulty.

We tested two neglect patients, one chronic, one recovered along with two RCVA control patients and 12 age-matched controls. Both neglect patients studied could successfully execute saccades into the neglected field when the target was presented alone. However, a dissociation emerged between the two patients when the target was presented with distractor items. Patient ERs first saccade to target performance in the three search conditions revealed clear effects of distractor type. In contrast for the recovered patient AF, the left/right difference was present for all search displays and appeared to be constant regardless of distractor type. This differential pattern of behaviour may reflect the different underlying neural causes of the neglect in these patients. In the current study, the measurement of saccades allowed the task to be fractionated, and thus, reveal the action of multiple mechanisms controlling saccades in search. (C) 2002 Elsevier Science Ltd. All rights reserved.
\end{abstract}

Keywords: Hemispatial neglect; Visual search; Eye movements; Target selection

\section{Introduction}

In order to explore the environment and obtain visual information, we constantly move our eyes. In this way, objects of interest are brought into the region of highest resolution, the fovea. The recording of eye movements in visual search tasks in which a designated target has to be detected among non-target distractors, thus, allows an in depth investigation of space exploration. Also, as eye movements are believed to reflect the cognitive processes that underlie search [32], their recording permits the investigation of such processes as well as the determination of factors that guide exploration. Eye movements have also been linked to attentional processes and their patterns can indicate how attention is allocated [16,22,26,47,50], but see also [31] for a different view. More importantly, as

\footnotetext{
* Corresponding author. Tel.: +44-141-330-6174; fax: +44-141-330-4606.

E-mail address: m.harvey@psy.gla.ac.uk (M. Harvey).
}

the current experiments demonstrate, the monitoring of eye movements can reveal abnormalities in search behaviour that are not apparent from reaction time (RT) data alone.

A number of studies have already shown that patients with hemispatial neglect are impaired in exploring the space on the side contralateral to their lesion, not only in terms of manual exploration, but also in relation to their eye movement patterns: typically, such patients demonstrate a rightward deviation of exploratory gaze [20,24,25], start their exploration in right hemispace and compared to healthy participants, spend relatively less time exploring the left $[3,4]$, but relatively more time exploring the right space [3]. In general, although being able to execute saccades to the left $[23,25]$, neglect patients execute fewer saccades into left space. However, if they do execute leftward saccades, these saccades are characterised by prolonged saccade latencies, hypometric amplitudes $[16,21,46]$ and a tendency to make multiple small saccades into left space [17].

Walker and Young [45] and Walker et al. [46] provided good evidence that the abnormal eye movement patterns of 
these patients are indeed a consequence of neglect and not its cause: their patient RR was able to initiate saccades to the left, but sometimes still failed to report the information presented on the left. In RRs case, the eye movement deficits could also not be attributed to a visual field defect, as this patient did not have any visual field cuts. In addition, Behrmann et al. [3] demonstrated that hemianopic neglect patients do not compensate for field cuts unlike other hemianopic subjects who, compared to healthy subjects, showed a higher proportion of fixations in the far left space. They argue that the gradient of attention in neglect causes an impoverished representation of visual space, which in turn leads to a pattern of eye movements which resembles the fundamental attentional deficit.

In fact, even simply looking at neglect patients' RT patterns in visual search tasks clearly shows an effect of side of presentation in that RTs are longer to contralesional than ipsilesional targets $[9,10,40]$. Interestingly, however, it has also been found that the contralesional slowing increases the more attentionally demanding the visual search tasks become $[1,9,10,17,40]$, pointing to the possible conclusion that pre-attentive processes may be much less affected in hemispatial neglect. However, pre-attentive processes were not completely unimpaired in the patients studied by Eglin et al. [9,10]. Although the patients' performance was better in the parallel compared to the serial search tasks, it was nonetheless impaired as RTs were longer when locating targets on the contralesional side whenever ipsilesional distractors were present.

In none of these visual search tasks were eye movements monitored. As a result, it is unclear what may have driven the differential RT performance. Eye movements were monitored in the search study by Behrmann et al. [3] who asked patients to report the number of A's in a random display of letters. As reported above neglect patients made fewer fixations and spent less time on the left of the display, whereas compared to the control group they made more and longer fixations on the right. In this study, the stimulus display was not altered, however.

The current study was designed to systematically investigate in what way an increase in display complexity and stimulus similarity influences saccadic as well as RT behaviour in neglect patients, and whether these two measures are always correlated.

In two pilot experiments on healthy participants, we have observed that the execution and direction of saccadic eye movements in visual search is influenced by low-level as well as higher-level factors. On a lower level, the execution of eye movements was shown to be affected by the mere presence of distractor stimuli: in a single target condition participants executed fast stimulus driven saccades that were triggered by the onset of the target stimuli. These saccades, however, were frequently suppressed in those conditions in which additional distractor letters were displayed. In these conditions, participants tended to maintain fixation in the centre of the display before responding. It was also found that the direction of saccades to distractor letters was clearly guided by the similarity of the distractors to the targets. Participants executed initial distractor-directed saccades significantly more frequently to a distractor that shared features with the target letters than to distractors that did not, thus, giving evidence for higher-level processes determining saccadic selection.

The main goal of the present experiment, was therefore, to investigate if these lower and higher-level effects are present in neglect patients and in the case of an impairment, if this is reflected in the RTs as well as in the saccadic patterns or if these factors dissociate.

Pierrot-Deseilligny et al. [38] and Gaymard et al. [14] have provided evidence that the cortex and specifically the parietal eye field (PEF) is involved in saccade-triggering. Lesions to the PEF can, thus, lead to impaired saccade generation and, as described previous, this has been found in neglect patients. In addition, saccadic latencies for leftward saccades may be prolonged, even for single isolated stimuli (see [18]). This suggests that saccade execution and latency may be impaired in neglect patients even in single target conditions.

Task difficulty and hereby attentional demands were increased over conditions by adding three distractor stimuli to the display, whose similarity with the target letters was systematically increased. It was expected that the patients' impairment would be more pronounced in the harder conditions both in terms of saccadic and RT performance.

\section{Method}

\subsection{Participants}

Two patients with hemispatial neglect (ER, AF), two patients with right hemisphere lesions, but no neglect (AQ, $\mathrm{MH}, \mathrm{RCVA}$ controls) and 12 healthy elderly participants matched in age and education were tested. All patients had normal or corrected to normal vision in both eyes and none of them suffered from oculomotor disorders.

In ERs case, re-testing before the experiment showed a BIT [48] score of 128/146. He showed the following neglect type errors: line crossing in the left half of page 18/18, right half of page $18 / 18$; letter cancellation left $15 / 20$, right 17/20; star cancellation left 19/27, right 26/27; line bisection $9 / 9$; figure and shape copying $3 / 4$ and representational drawing $3 / 3$. AF, however, who had previously exhibited neglect, showed an improved score of 136/146 at re-testing which would classify him as a recovered neglect patient. Nonetheless, he still showed neglect type errors in the BIT: line crossing left half of page 18/18, right half of page 18/18; letter cancellation left $18 / 20$, right $16 / 20$; star cancellation left 25/27, right 27/27; line bisection 8/9; figure and shape copying $3 / 4$ and representational drawing $3 / 3$.

Although the RCVA control patient MH showed omissions on the BIT, these were not lateralised and occurred only for the cancellation, but not the copying tasks 
Table 1

Participant information

\begin{tabular}{|c|c|c|c|c|c|}
\hline & \multirow[t]{2}{*}{ Controls } & \multicolumn{2}{|l|}{ Neglect patients } & \multicolumn{2}{|l|}{ RCVA controls } \\
\hline & & ER & $\mathrm{AF}$ & $\mathrm{AQ}$ & $\mathrm{MH}$ \\
\hline $\operatorname{Age}^{a}$ & $72.7(4)$ & 77 & 63 & 66 & 75 \\
\hline Sex & Male, female & Male & Male & Male & Male \\
\hline Education $^{\mathrm{a}}$ & $14(3.8)$ & 12 & 13 & 15 & 9 \\
\hline Handedness & Right & Right & Right & Right & Right \\
\hline Lesion location & - & Right fronto-temporal & Right thalamus & Right fronto-parietal & Right fronto-parietal \\
\hline Time since onset & - & 30 months & 12 months & 15 months & $\begin{array}{l}\text { Three strokes: } 60,36 \\
\text { and } 13 \text { months }\end{array}$ \\
\hline Hemianopia & - & No & No & No & No \\
\hline Hemiparesis & - & Yes & Yes & Yes & Yes \\
\hline Extinction & - & No & Yes & No & No \\
\hline BIT after stroke onset & - & $116^{\mathrm{b}}$ & $68^{\mathrm{b}}$ & $144^{\mathrm{b}}$ & $142^{\mathrm{b}}$ \\
\hline BIT re-testing before experiment & - & $128^{\mathrm{b}}$ & $136^{\mathrm{b}}$ & $144^{\mathrm{b}}$ & $144^{\mathrm{b}}$ \\
\hline
\end{tabular}

${ }^{\text {a }}$ Given in mean (S.D.) in years.

${ }^{\mathrm{b}}$ Maximum score, 146; cut-off, 129.

AQ

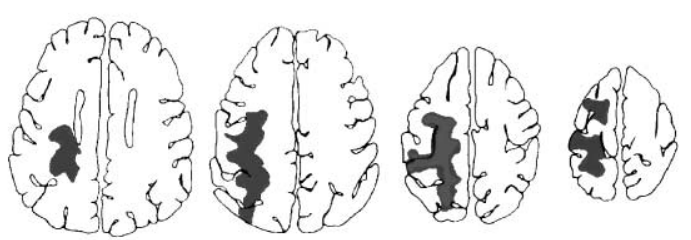

MH

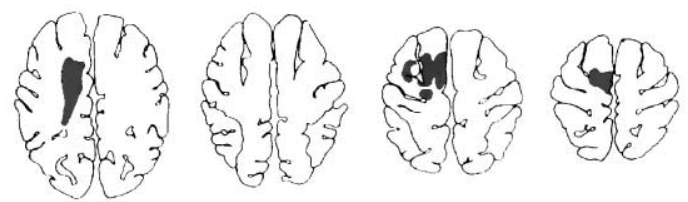

ER

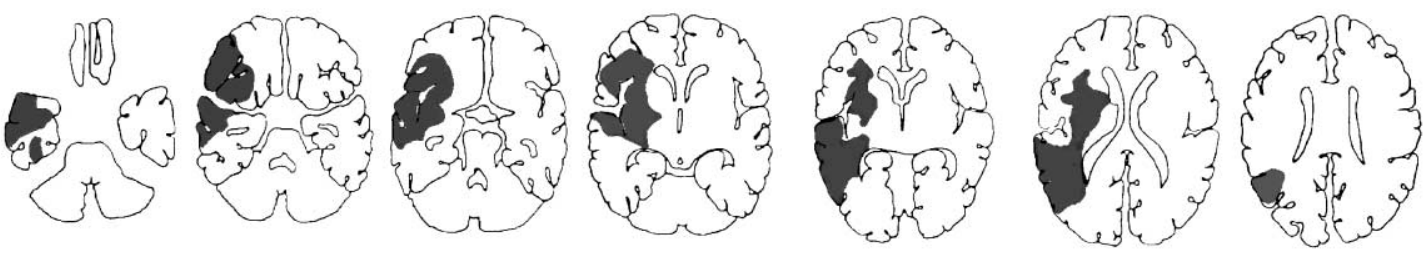

AF

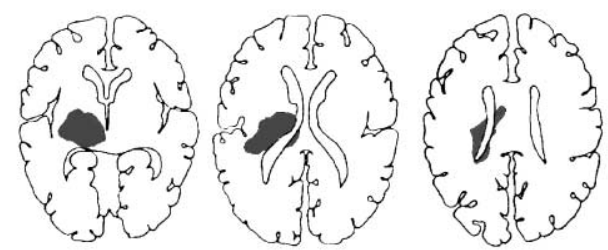

Fig. 1. Individual CT maps of damaged areas of the four patients. The figure shows for AQ slices, $8-11$ (at $0^{\circ}$ ); for $\mathrm{MH}$ slices, 10,12 and 13 (at $5^{\circ}$ ); for ER slices, 1-7 (at 20 ); and for AF slices, 5-7 (at 20 ${ }^{\circ}$ ). Templates were taken from [6].

(see Table 1 for details of all participants and Fig. 1 for lesion mapping ${ }^{1}$ ).

\footnotetext{
${ }^{1}$ ERs lesion was initially diagnosed by a neuroradiologist to be in the right parietal lobe. However, subsequent more precise mapping revealed that his lesion was fronto-temporal (see Fig. 1). Unfortunately, this incorrect information is given in [35] and [36].
}

\subsection{Displays}

Displays were generated and presented on an IBM compatible personal computer with a $17^{\prime \prime}$ VGA monitor (133 MHz Compaq Deskpro). The display consisted of four capital letters arranged symmetrically in a square around the fixation point $\left(4^{\circ}\right.$ to the left or right of fixation and 
displaced $4^{\circ}$ vertically above or below the horizontal axis of fixation). The fixation point was a white circle on black background with a diameter of $0.5^{\circ}$. The letter stimuli were written in uppercase (Arial) and subtended $1.2^{\circ}$ of visual angle horizontally and $1.4^{\circ}$ vertically. They appeared white on a black background. The target was the letter $\mathrm{T}$ in half of the trials and the letter $\mathrm{H}$ in the other half. A target was present in every trial and appeared equally often in all four possible locations. There were 48 trials in a block, 24 with each target type. When three distractor types were present, the location of the distractors was fully counterbalanced. The distance between eyes and screen was $57 \mathrm{~cm}$. Example displays are shown in Fig. 2.
The experiment consisted of four conditions. In condition 1 , the target letter ( $\mathrm{T}$ or $\mathrm{H}$ ) was displayed on its own without any distractors. In condition 2 , the target letter was displayed with three capital letter $\mathrm{O}$ distractors. In condition 3 , the heterogeneous distractors $\mathrm{S}, \mathrm{O}$ and $\mathrm{U}$ and in condition 4 , the distractors A, X, Y were used.

Previous pilot work demonstrated that the letter $\mathrm{U}$ was more similar to the target letters than the letters $\mathrm{S}$ or $\mathrm{O}$. Letters $\mathrm{A}, \mathrm{X}$ and $\mathrm{Y}$ were found to be of equal similarity to the target letters [34].

Participants viewed the displays with both eyes, but only data of the eye with the best spatial accuracy was analysed.

Condition 1
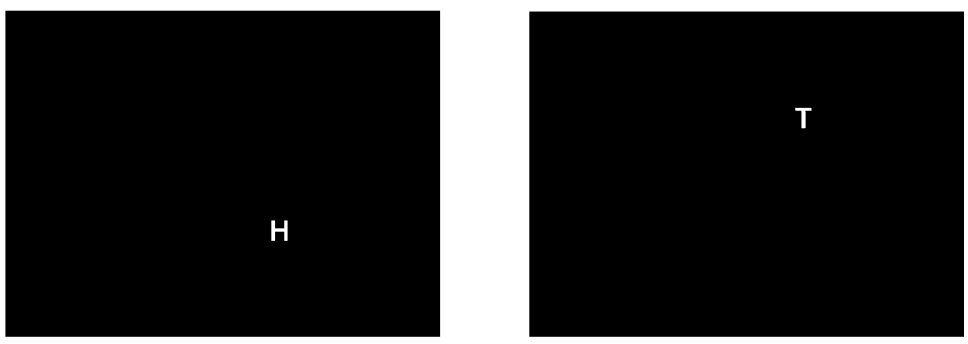

Condition 2
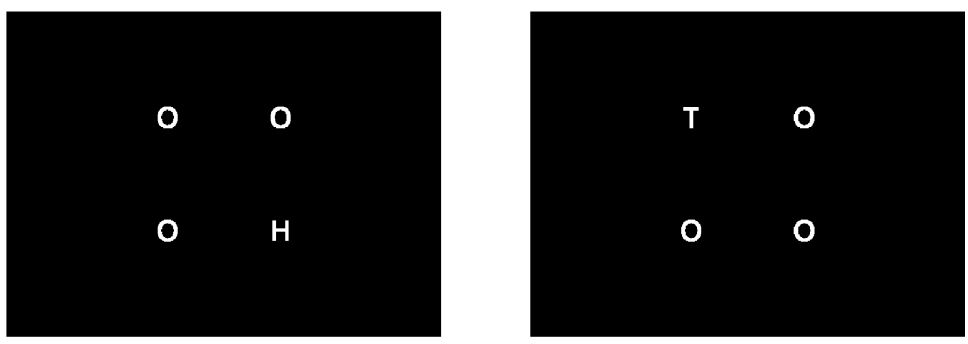

Condition 3
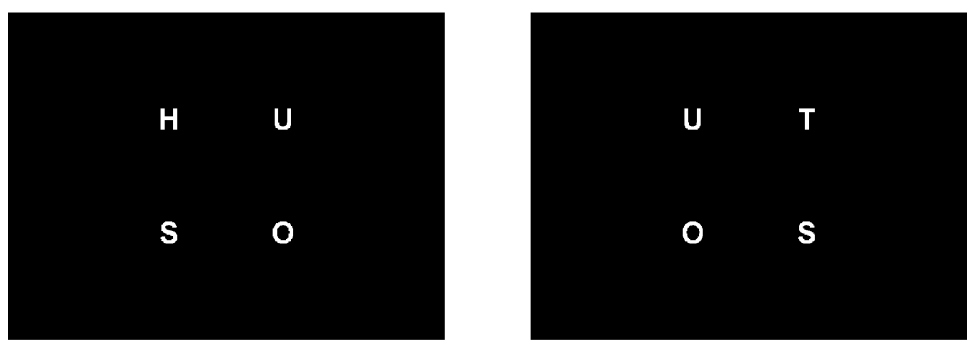

Condition 4
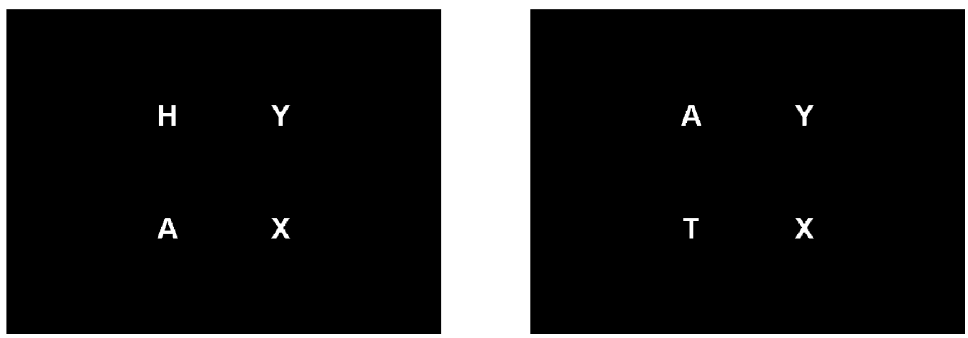

Fig. 2. Example displays with the target letters H (left-hand column) or T (right-hand column). 


\subsection{Procedure}

The fixation point was presented until fixation was stable (as assessed by the experimenter) and disappeared with stimulus presentation. Stimuli remained visible until participants pressed one of two response buttons positioned one above the other in front of them. Participants, whose heads rested in a chin rest, were instructed to decide whether either the target letter $\mathrm{T}$ or $\mathrm{H}$ was present in the display and press one of the response buttons. Half of the healthy participants pressed the upper button for $\mathrm{T}$ and the lower button for $\mathrm{H}$, the other half responded vice versa. The order of conditions was counterbalanced between control participants.

Longer breaks between conditions were needed during the testing of the patients. In addition, each patient completed each experimental condition twice so that there were 96 trials per condition. Healthy controls performed 48 trials per condition.

\subsection{Eye movement recording and analysis}

Eye movements were recorded with the SMI EyeLink System (SensoMotoric Instruments GmbH, Teltow, Germany) using the centre of the pupil as well as the corneal reflection technique to define pupil position.

Each block of trials was preceded by a nine-point grid calibration and validation for which participants were instructed to saccade to a white circle $\left(0.5^{\circ}\right)$ on a black background, which appeared sequentially at nine points in a square array. Between trials, the fixation circle reappeared to correct for drift due to head movements. Eye movements were recorded at $250 \mathrm{~Hz}$ sample rate at a spatial resolution, typically, of $0.3^{\circ}$. Saccade onset was defined as a change in eye position with a minimum velocity of $30^{\circ} \mathrm{s}$ or a minimum acceleration of $8000^{\circ} / \mathrm{s}^{2}$. Saccades were considered to be in the direction of a stimulus when their amplitude in that direction was larger than $0.5^{\circ}$.

In the subsequent data analysis, trials were rejected if the initial fixation location at the start of the trials was not within $0.5^{\circ}$ of the centre of the fixation point. This was a strict criteria and led to the exclusion of a significant proportion of the subsequently analysed data. However, such a criteria insures that off-centre fixation does not in itself bias saccading to one side or the other. In addition, trials with a saccade latency shorter than $100 \mathrm{~ms}$ and trials with manual reaction times shorter than $100 \mathrm{~ms}$ or indeed with incorrect manual responses were excluded. Trials with saccades with an amplitude smaller than $1^{\circ}$ and larger than $10.3^{\circ}$ were discarded.

\section{Results}

\subsection{Analyses}

The data was analysed separately for each patient and compared to the performance of the healthy controls. There were five dependent measures in the current study. These were the manual reaction time and properties of the first saccade. Only the first saccade was analysed as it is only for the programming of this saccade that the stimulus display contains items matched in both the left and the right visual fields.

The following measures were taken and the following analyses performed.

Manual RTs of the healthy control group were analysed with a $4 \times 2 \times 2$ ANOVA with repeated measures with the factors conditions (1-4), target location left/right and target location top/bottom. For the patients, a general factorial ANOVA was carried out with the same factors.

Saccade latencies and saccade amplitudes of the first saccades were also measured and analysed for the control group with ANOVAs with repeated measures with the factors conditions (1-4), saccade direction left/right and saccade direction top/bottom. The patient data was analysed for the same factors with general factorial ANOVAs.

The percentage of target-directed first saccades was analysed with an ANOVA with repeated measures for the controls with the factors conditions (2-4), target location left/right and target location top/bottom. The number of correct saccades were analysed for the patients with loglinear analyses with the factors conditions (2-4), correct saccade (correct versus incorrect), target location left/right and target location top/bottom. Saccades were considered correct if they passed the exclusion criteria as outlined above and were directed into the quadrant containing the target letter. Condition 1 was excluded from these analyses as performance was at ceiling for all participants.

The distribution of distractor-directed first saccades among distractor letters was analysed separately for conditions 3 and 4 with repeated measures ANOVAs with the factor letter for the controls, and with $\chi^{2}$-tests for the patient data. Saccades were scored on the basis of which quadrant they were directed towards.

Although it would have been interesting to further split these distractor-directed first saccades into those directed towards the right and leftward locations this was not possible as the number of saccades proved too small (N's less than 10) to allow a meaningful interpretation. Also, although the factor top/bottom was analysed throughout, for brevity the results will not be reported here as they are not relevant.

Table 2 shows the percentages of trials which were discarded from further analysis mainly due to the poor fixations the participants showed.

\subsubsection{Manual reaction time (RT)}

For the healthy subjects, there was a significant effect of condition with RTs increasing from conditions 1-4. Post-hoc Newman-Keuls tests showed significant differences between conditions 1 and 3 and 1 and 4 . (see Table 3 for details and effect sizes).

This pattern was mirrored in the RCVA control patient AQ: there was a significant effect of condition.

For the RCVA control patient MH, there was also a significant effect of condition with shorter RTs in conditions 1 
Table 2

Percentage of discarded trials for each patient and the control group

\begin{tabular}{lllllrr}
\hline & Poor fixation & Latency & Amplitude & RT & Manual errors & Recording errors \\
\hline Controls & 21.61 & 4.77 & 3.99 & 0 & 1.97 & 0.09 \\
ER & 25.78 & 3.65 & 0.52 & 0 & 0.26 & 0 \\
AF & 25.78 & 5.21 & 0.26 & 0.3 & 0.04 & 0.52 \\
MH & 23.96 & 1.56 & 3.39 & 0 & 0 & 0.21 \\
AQ & 13.80 & 2.60 & 0.26 & 0 & 32.55 \\
\hline
\end{tabular}

and 2 than in conditions 3 and 4. RTs were also significantly shorter when targets appeared on the right compared to the left side.

The data of the neglect patient ER, apart from showing an effect of condition and target location left/right also revealed an interaction of condition $\times$ target location left/right, reflecting differences in RTs between targets on the left and on the right between the conditions. Planned comparisons showed that the difference was very small and non-significant in condition 1 , but was significant in all other conditions with a maximum difference of $173 \mathrm{~ms}$ in condition 4 .

Neglect patient AFs data also showed significant condition and target location left/right effects in that RTs were fastest in condition 1 . There were also significant differences between conditions 2 and 4 and 3 and 4 as revealed by Newman-Keuls post-hoc tests. However, there was no interaction indicating that the slowed responses towards leftwardly located targets were not modulated by task difficulty.

\subsubsection{Latencies of first saccades}

For the healthy subjects only the factor saccade direction left/right was significant with leftward saccades being initiated faster than rightward saccades (see Table 3 for details and effect sizes).

The RCVA control patient AQ also initiated leftward saccades significantly faster than rightwards saccades. In his case, the factor condition was also significant with shortest latencies in condition 1.

For MH (RCVA control), only the factor condition reached significance. Post-hoc Newman-Keuls revealed that saccade latencies were significantly shorter in condition 1 than in the other three conditions.

The neglect patient ER showed significant effects of condition and saccade direction left/right as well as a significant interaction indicating a difference in the magnitude of the left/right asymmetry with conditions. Planned comparisons showed that rightward saccades were initiated significantly faster than leftward saccades for all four conditions.

The neglect patient AF showed an effect of saccade direction only, with shorter rightwards than leftwards saccades throughout.

\subsubsection{Amplitudes of first saccades}

For the healthy subjects saccade amplitude was not altered by any of the manipulations. (see Table 3 for details and effect sizes).
For the RCVA control patient AQ, saccade amplitude was altered by condition as well as target location left/right, rightward saccades being longer overall than leftward saccades. The interaction was also significant. Planned comparisons showed reliable left/right differences for conditions 1 and 2 only.

The control patient $\mathrm{MH}$ as well as the two neglect patients (ER, AF) all showed significant effects of condition with largest amplitudes for condition 1 and a significant target location effect with shorter saccades for leftward than rightward located targets.

It is worth noting that the control patient $\mathrm{MH}$ executed saccades in only $40 \%$ of all trials, a behaviour very different from all the other patients who executed saccades on almost every trial (AQ: 98.5\%, ER: 91\%, AF: 100\%) and the healthy controls who executed saccades on $68 \%$ of trials.

\subsubsection{Percentage of first saccades made to target}

For the healthy control subjects, condition had a significant effect on the percentage of correct saccades $(F(2,6)=$ $11.8, P<0.01)$ with post-hoc Newman-Keuls tests showing differences between conditions 2 and 3 and conditions 3 and 4 (analysed for conditions 2 to 4 only).

Tests of partial association showed the same result for the RCVA control AQ. The interaction condition $\times$ correct saccades reached significance $\left(\chi^{2}(2)=13.2, P=0.001\right)$, again indicating that the percentage of correct saccades decreased with difficulty.

For the RCVA control patient MH, tests of partial association revealed no significant effects.

For the neglect patient ER, a significant interaction for condition $\times$ correct saccades $\left(\chi^{2}(2)=13.7, P<0.01\right)$ was found as well as an interaction between target location left/right $\times$ correct saccade $\left(\chi^{2}(1)=30.5, P<0.01\right)$. Interestingly, however, the difference between right and left correct saccades increased as task difficulty increased over conditions (interaction of condition $\times$ correct saccade $\times$ target location left/right $\left(\chi^{2}(2)=11.2, P=0.03\right)$ : the number of his correct leftward saccades decreased over conditions. When the target appeared on the right side though, his performance was relatively constant.

In contrast to $\mathrm{ER}, \mathrm{AFs}$ neglect data revealed an interaction of correct saccade $\times$ target location left/right only $\left(\chi^{2}(1)=\right.$ 45.2, $P<0.01)$. More correct saccades were made when the target appeared on the right side. So in contrast to ERs 
Table 3

Means for reaction time, saccade latencies and amplitudes, presented for left and right target location, for each condition (effect sizes for the main effects of condition (Condition) and target location left/right (Target location) as well as for the interaction are also given)

\begin{tabular}{|c|c|c|c|c|c|c|c|c|c|c|}
\hline \multirow[t]{3}{*}{ Condition } & \multicolumn{10}{|c|}{ Reaction time (ms) } \\
\hline & \multicolumn{2}{|l|}{ Control group } & \multicolumn{2}{|l|}{ MH } & \multicolumn{2}{|l|}{$\mathrm{AQ}$} & \multicolumn{2}{|l|}{ ER } & \multicolumn{2}{|l|}{$\mathrm{AF}$} \\
\hline & Left & Right & Left & Right & Left & Right & Left & Right & Left & Right \\
\hline \multicolumn{11}{|c|}{ Target location } \\
\hline 1 & 638 & 633 & 607 & 548 & 641 & 650 & 645 & 628 & 834 & 692 \\
\hline 2 & 714 & 719 & 607 & 652 & 633 & 633 & 749 & 646 & 771 & 650 \\
\hline 3 & 754 & 738 & 842 & 772 & 780 & 854 & 799 & 636 & 876 & 906 \\
\hline 4 & 836 & 795 & 847 & 687 & 983 & 932 & 890 & 717 & 1129 & 931 \\
\hline Condition & $\begin{array}{l}F(3,21)=7.52 \\
P<0.01\end{array}$ & $\begin{array}{l}F(3,21)=7.52 \\
P<0.01\end{array}$ & $\begin{array}{l}F(3,255)=25.2 \\
P<0.01\end{array}$ & $\begin{array}{l}F(3,255)=25.2 \\
P<0.01\end{array}$ & $\begin{array}{l}F(3,248)=3.2 \\
P<0.01\end{array}$ & $\begin{array}{l}F(3,248)=3.2 \\
P<0.01\end{array}$ & $\begin{array}{l}F(3,252)=14.0 \\
P<0.01\end{array}$ & $\begin{array}{l}F(3,252)=14.0 \\
P<0.01\end{array}$ & $\begin{array}{l}F(3,243)=16.7 \\
P<0.01\end{array}$ & $\begin{array}{l}F(3,243)=16.7 \\
P<0.01\end{array}$ \\
\hline $\begin{array}{l}\text { Target } \\
\text { location }\end{array}$ & N.S. & N.S. & $\begin{array}{l}F(1,255)=14.1 \\
P<0.01\end{array}$ & $\begin{array}{l}F(1,255)=14.1 \\
P<0.01\end{array}$ & N.S. & N.S. & $\begin{array}{l}F(1,252)=35.2 \\
P<0.01\end{array}$ & $\begin{array}{l}F(1,252)=35.2 \\
P<0.01\end{array}$ & $\begin{array}{l}F(1,243)=9.29 \\
P<0.01\end{array}$ & $\begin{array}{l}F(1,243)=9.29 \\
P<0.01\end{array}$ \\
\hline Interaction & N.S. & N.S. & N.S. & N.S. & N.S. & N.S. & $\begin{array}{l}F(3,252)=3.45 \\
P=0.02\end{array}$ & $\begin{array}{l}F(3,252)=3.45 \\
P=0.02\end{array}$ & N.S. & N.S. \\
\hline & \multicolumn{10}{|c|}{ Saccade latencies (ms) } \\
\hline \multicolumn{11}{|c|}{$\overline{\text { Saccade direction }}$} \\
\hline 1 & 198 & 223 & 204 & 222 & 172 & 186 & 203 & 157 & 243 & 140 \\
\hline 2 & 272 & 337 & 343 & 311 & 198 & 227 & 313 & 194 & 244 & 177 \\
\hline 3 & 284 & 306 & 314 & 297 & 219 & 246 & 367 & 209 & 261 & 177 \\
\hline 4 & 263 & 315 & 345 & 242 & 211 & 240 & 301 & 187 & 259 & 171 \\
\hline Condition & N.S. & N.S. & $\begin{array}{l}F(3,91)=5.12 \\
P<0.01\end{array}$ & $\begin{array}{l}F(3,91)=5.12 \\
P<0.01\end{array}$ & $\begin{array}{l}F(3,248)=21.0 \\
P<0.01\end{array}$ & $\begin{array}{l}F(3,248)=21.0 \\
P<0.01\end{array}$ & $\begin{array}{l}F(3,228)=19.1 \\
P<0.01\end{array}$ & $\begin{array}{l}F(3,228)=19.1 \\
P<0.01\end{array}$ & N.S. & N.S. \\
\hline $\begin{array}{l}\text { Target } \\
\text { location }\end{array}$ & $\begin{array}{l}F(1,4)=10.8 \\
P=0.03\end{array}$ & $\begin{array}{l}F(1,4)=10.8 \\
P=0.03\end{array}$ & N.S. & N.S. & $\begin{array}{l}F(1,248)=14.4 \\
P<0.01\end{array}$ & $\begin{array}{l}F(1,248)=14.4 \\
P<0.01\end{array}$ & $\begin{array}{l}F(1,228)=69.8 \\
P<0.01\end{array}$ & $\begin{array}{l}F(1,228)=69.8 \\
P<0.01\end{array}$ & $\begin{array}{l}F(1,243)=51.3 \\
P<0.01\end{array}$ & $\begin{array}{l}F(1,243)=51.3 \\
P<0.01\end{array}$ \\
\hline Interaction & N.S. & N.S. & N.S. & N.S. & N.S. & N.S. & $\begin{array}{l}F(3,228)=4.17 \\
P<0.01\end{array}$ & $\begin{array}{l}F(3,228)=4.17 \\
P<0.01\end{array}$ & N.S. & N.S. \\
\hline & \multicolumn{10}{|l|}{ Amplitudes $\left({ }^{\circ}\right)$} \\
\hline \multicolumn{11}{|c|}{ Saccade direction } \\
\hline 1 & 5.25 & 5.51 & 4.79 & 4.81 & 6.81 & 8.3 & 4.89 & 5.09 & 4.06 & 4.69 \\
\hline 2 & 4.56 & 4.22 & 2.62 & 5.38 & 5.58 & 7.29 & 4.31 & 4.68 & 3.89 & 3.9 \\
\hline 3 & 4.20 & 4.62 & 3.82 & 4.76 & 5.9 & 6.55 & 3.89 & 4.12 & 3.32 & 3.47 \\
\hline 4 & 4.26 & 4.39 & 3.03 & 4.47 & 5.37 & 5.96 & 4.18 & 4.43 & 3.37 & 3.68 \\
\hline Condition & N.S. & N.S. & $\begin{array}{l}F(3,91)=3.7 \\
P=0.01\end{array}$ & $\begin{array}{l}F(3,91)=3.7 \\
P=0.01\end{array}$ & $\begin{array}{l}F(3,248)=33.3 \\
P<0.01\end{array}$ & $\begin{array}{l}F(3,248)=33.3 \\
P<0.01\end{array}$ & $\begin{array}{l}F(3,228)=6.6 \\
P<0.01\end{array}$ & $\begin{array}{l}F(3,228)=6.6 \\
P<0.01\end{array}$ & $\begin{array}{l}F(3,243)=7.9 \\
P<0.01\end{array}$ & $\begin{array}{l}F(3,243)=7.9 \\
P<0.01\end{array}$ \\
\hline $\begin{array}{l}\text { Target } \\
\text { location }\end{array}$ & N.S. & N.S. & $\begin{array}{l}F(1,91)=10.3 \\
P<0.01\end{array}$ & $\begin{array}{l}F(1,91)=10.3 \\
P<0.01\end{array}$ & $\begin{array}{l}F(1,248)=41.5 \\
P<0.01\end{array}$ & $\begin{array}{l}F(1,248)=41.5 \\
P<0.01\end{array}$ & $\begin{array}{l}F(1,228)=7.5 \\
P=0.01\end{array}$ & $\begin{array}{l}F(1,228)=7.5 \\
P=0.01\end{array}$ & $\begin{array}{l}F(1,243)=6.4 \\
P=0.01\end{array}$ & $\begin{array}{l}F(1,243)=6.4 \\
P=0.01\end{array}$ \\
\hline Interaction & N.S. & N.S. & N.S. & N.S. & $\begin{array}{l}F(3,248)=3.54 \\
P=0.02\end{array}$ & $\begin{array}{l}F(3,248)=3.54 \\
P=0.02\end{array}$ & N.S. & N.S. & N.S. & N.S. \\
\hline
\end{tabular}



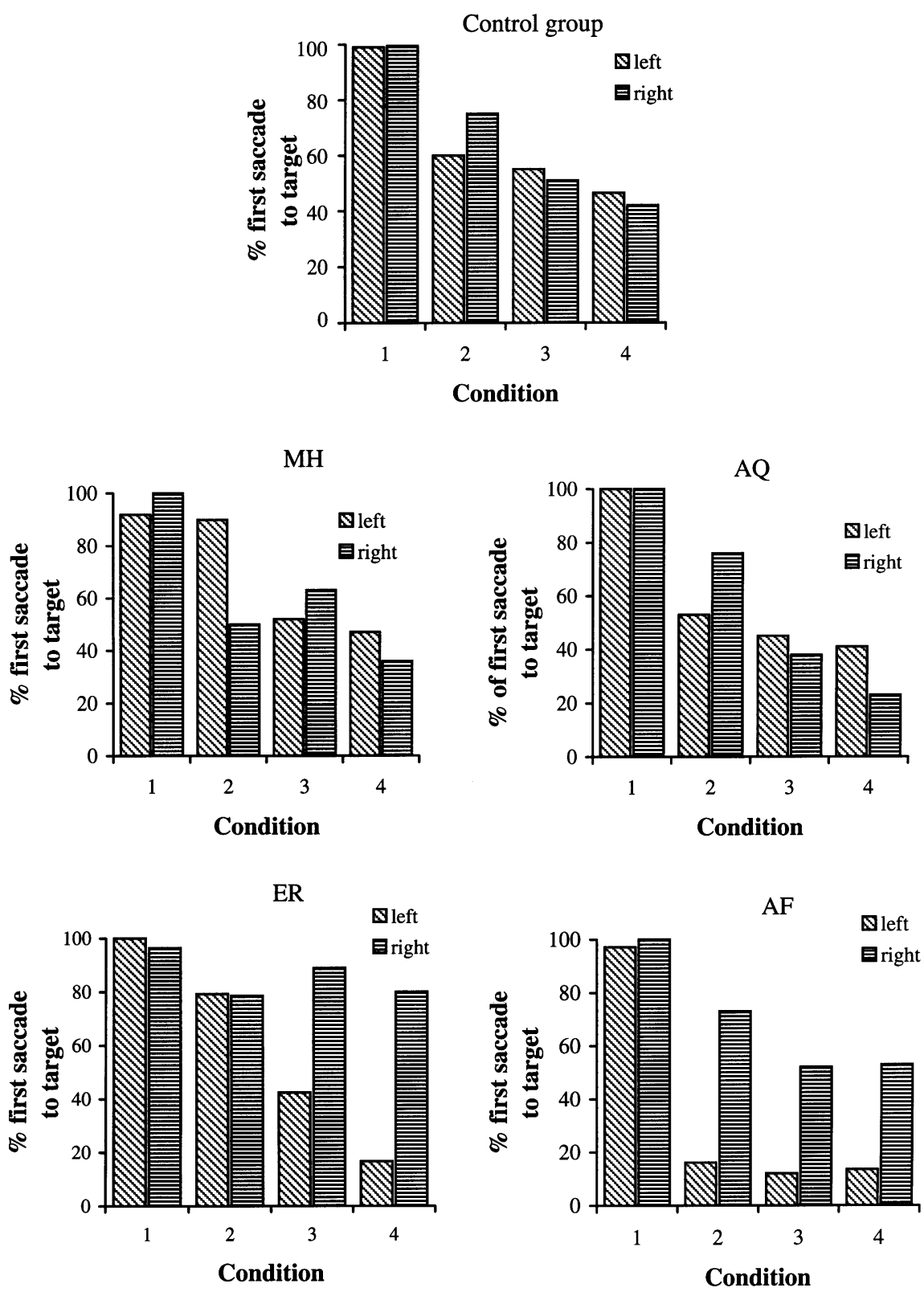

Fig. 3. Percentage of first saccades to target with regard to target location left or right. Separate graphs are presented for the four patients and another graph averaged across the control subjects. Error bars on the control data indicate standard errors.

performance, AF showed no differential effect of task difficulty in search conditions $2-4$.

The percentages of correct saccades with regard to target location for all subjects are shown in Fig. 3.

\subsubsection{Distribution of distractor-directed first saccades over distractor letters}

The healthy subjects showed that in condition 3 significantly more incorrect saccades were directed to the distractor $\mathrm{U}$ than the $\mathrm{O}$ and $\mathrm{S}(F(2,22)=6.19, P=0.007)$. In condition 4 , incorrect saccades were distributed evenly over the three distractors. These effects were also found for the RCVA control patient AQ $\left(\chi^{2}(2)=8.47, P=0.01\right)$ and the neglect patient ER $\left(\chi^{2}(2)=12.1, P=0.002\right)$. The RCVA control MH showed a trend in this direction which did not reach significance. The neglect patient AF, however, showed no tendency to guide his eye movements towards the more similar distractor $U$. The results of all subjects are shown in Fig. 4.

\subsection{Summary of the main results}

As the motivation of this study was to investigate the effects of hemispatial neglect on eye movement and RT patterns in visual search, the discussion will contrast the performance of the two neglect patients with the RCVA and 


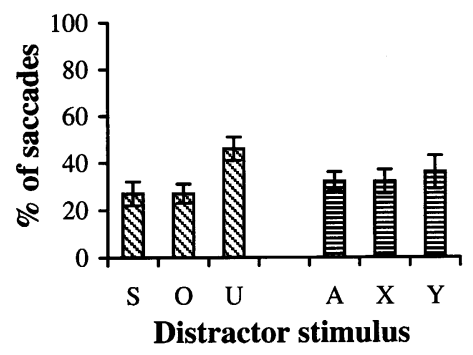

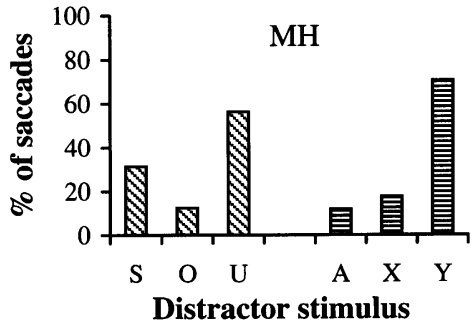

ER

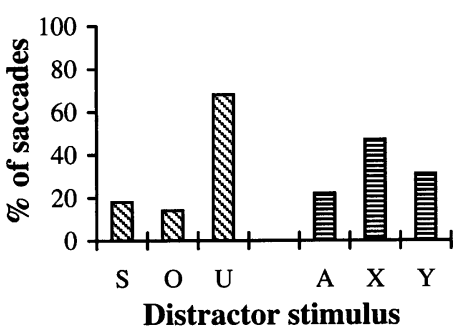

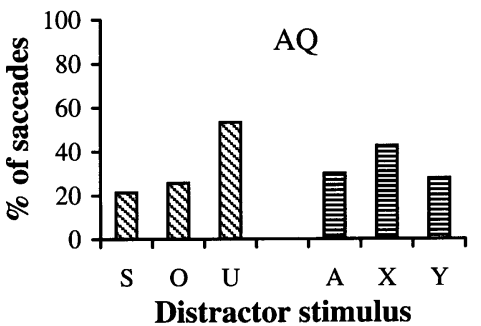

$\mathrm{AF}$

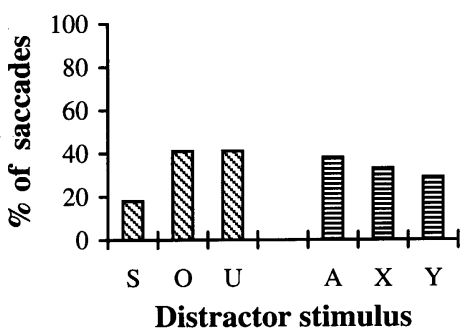

Fig. 4. Distribution of non-target directed first saccades in conditions 2 and 3. The numbers indicate the percentage of non-target directed first saccades. Separate graphs are presented for the four patients and another graph averaged across the control subjects. Error bars on the control data indicate standard errors.

healthy controls rather than give an in depth analysis of the control data as such.

In terms of the manual RT data, both neglect patients showed clear left/right asymmetries: their responses were slowed towards leftwardly located targets for all experimental conditions (with the exception of condition 1 for ER), a pattern not seen in the healthy control subject data and $\mathrm{AQ}$, although the RCVA control patient $\mathrm{MH}$ also showed this result. This asymmetry was still apparent in the saccade latencies and in fact directly contrasted with the data of the healthy control subjects and RCVA control AQ who executed saccades towards leftwardly located targets faster than saccades towards targets on the right (control patient MH did not show a significant lateral saccade latency bias).

For the target directed first saccades clear differences emerged between the two neglect patients. For AF, there were clear left/right differences for all conditions in which multiple elements were presented (conditions 2-4) and the left/right cost was constant across these conditions. In contrast for ER, there was no left/right cost for the displays that contained a single item (condition 1) or easy search (condi- tion 2), but a cost was present in the more difficult search conditions (conditions 3 and 4).

ER also showed clear evidence for stimulus guidance effects: just like the controls, in condition 3, he looked significantly more often to the similar distractor letter (U) than to the more dissimilar distractors letters (O and $\mathrm{S})$. Patient AF showed no such stimulus guidance. None of the controls showed left/right effects for percentage of correct saccades and all, but $\mathrm{MH}$ showed evidence of stimulus guidance for condition 3 (for $\mathrm{MH}$, there was only a non-significant trend).

\section{Discussion}

The objective of the present study was to investigate if and how the effects of display complexity and stimulus similarity influence saccadic as well as RT behaviour in neglect patients.

For the single item display, like the control subjects' behaviour, almost $100 \%$ of the patients' first saccades were executed towards the target and there was no left/right 
asymmetry. For ER, there was also no asymmetry in terms of his manual reaction times for single targets on the left or right, however, there was a small difference for AF. In contrast, both patients showed left/right asymmetries in terms of their saccade latencies. Over all conditions, when compared to the control subjects, saccade latencies towards rightwardly located targets were executed faster, whereas leftward saccades had approximately the same latencies as those of the controls. These data indicate that both neglect patients were able to generate saccades towards targets in either left or right space, but did show a latency based asymmetry favouring the ipsilesional side. This complements a pattern found by Làdavas et al. [30] in a reaction time study. They showed that patients detected targets more poorly in the relative left than right of two horizontally arranged boxes, but detected the rightmost targets significantly faster than controls, indicating a stronger bias to allocate attention to the most ipsilesional items. This RT data is mimicked in the saccade latencies and increased number of correct saccades reported here. Neglect clearly has an oculomotor impact for both simple saccade generation and more complex search-based saccades. This is reflected in the latency asymmetries in the single as well as the multi-target conditions. In fact, the shorter rightward latencies can be explained with the saccade generation model put forward by Findlay and Walker [13]. Within this model, neglect is a consequence of an imbalance in the system that affects the automated processes in the spatial channel on the same side as the brain damage. They propose that the ipsilesional automatic peripheral detection processes (involved in orienting to contralesional stimuli) remain permanently underactivated. As a result the salience map involved in coding saccade metrics remains permanently depressed, making it unlikely that a peak will occur on the contralesional side. This would result in the relatively faster rightward saccades generated here.

In all the multi-element search displays (conditions 2-4) both neglect patients produced prolonged manual reaction times towards leftwardly located stimuli. This pattern of behaviour has already been reported in other visual search studies $[9,10,40]$, however, only in patient ER did the contralesional slowing increase systematically the more difficult the task became (see $[1,9,10,17,40]$ ). Simply taking the manual RT data into account also seems to suggest that performance in condition 2 may not have been entirely unimpaired. Similarly to the Eglin et al. $[9,10]$ studies, although the two patients' performance was better in the easier search task compared to the other conditions, it was nonetheless impaired in that manual RTs were longer when locating targets on the contralesional side whenever ipsilesional distractors were present (condition 2). This was not the case for condition 1.

Analyses of the first saccades, however, allowed far more detailed insight into the patients' behaviour. The two neglect patients differed in their saccadic performance in two distinct respects.
A number of studies have shown that non-target directed first saccades are significantly more likely to land on distractors sharing a target feature; this is visual guidance of the saccade [11]. This effect is closely related to the properties of Wolfe's guided search model [49]. Looking at guidance, ERs first saccade to target performance in condition 3 revealed clear effects of distractor type. Like both sets of controls he was more likely to make saccades to a distractor that was similar rather than dissimilar to the target. In contrast AF showed no such similarity effect.

Secondly, the two neglect patients differed in the extent to which the severity of their neglect was modulated by the nature of the display. AF showed highly asymmetric first saccades in condition 2 which remained constant across the three search conditions. In contrast ERs first saccades to target were virtually normal in condition 2 with no leftward bias, a bias then emerged in condition 3 which became even greater in condition 4 . Both sets of control groups showed no left/right asymmetries in their first saccades to target suggesting that these patterns of deficits are restricted to patients with hemispatial neglect. It is possible that the search asymmetry shown by AF may reflect a systematic search strategy adopted for all conditions. However, such an explanation seems unlikely for two reasons. Firstly, there is no asymmetry in condition 1 and secondly there are differences between conditions two and three and four although these are small. Nonetheless, AF was the only patient showing leftward extinction and it is possible that this resulted in the biases found for conditions two to four. Although $\mathrm{AF}$ never failed to press the response button for a leftward target stimulus, it is possible that his extinction biased saccadic performance rightwards whenever letters were presented on both sides of the visual field (conditions 2-4).

The differences these two patients showed in terms of their saccadic performance, despite fairly similar reaction time patterns, clearly demonstrates that studying saccades in this manner allows a far more detailed insight into the search mechanisms employed by the patients: apart from the most difficult condition 4, ERs visual search behaviour was still highly efficient with a gradual deterioration the more difficult the task became. AF on the other hand already produced highly inefficient saccadic searches in the simple condition 2. Liversedge and Findlay [32] have argued that eye movements reflect the cognitive processes that underlie search and a tight relationship between attentional orienting and saccade programming has indeed often been pointed out $[8,12,15,19,26,27,39,41,50]$. It, thus, seems likely that, despite his biased manual reaction times, which were similar to AFs, ER still processed even the leftward parts of the stimulus display to a much higher degree than AF.

The current experiment also demonstrates that there can be dissociations between RT and saccadic performance and that the parameters may well be decorrelated: in ERs case, his saccadic search behaviour turned out to be much better than predicted from the RT patterns whereas in AFs case it turned out to be much worse. This is particularly inter- 
esting as AF was judged to have recovered from neglect (BIT performance) whereas ER still had chronic neglect, yet he showed the better search performance. It would be interesting to assess if ERs better search performance may have been apparent on other clinical measures such as neglect awareness or Barthel functional living score, however, such measurements were not taken in the current study.

The differences between the two patients in terms of their saccadic performance may be accounted for by the varying lesion sites although any interpretation in terms of lesions site has to be highly speculative due to the single case nature of this study. Nonetheless, a TMS study run by Ashbridge et al. [2] found that applying magnetic stimulation over the right parietal cortex of subjects had no detrimental effects on performance in the pop-out search, but resulted in significantly impaired conjunction search. However, both of our right hemisphere controls had right parietal damage without showing impaired conjunction search and ER who did, had a fronto-temporal lesion. It is possible that the parietal sub-regions supposedly mediating visual search were spared in the control patients and that ERs lesion did extend into this area despite the lesion mapping not showing this. Alternatively, the temporal cortex has been implicated in visual search. Recording from single cells, Chelazzi and co-workers $([5,7])$ found evidence that networks in the inferior temporal cortex may select the visual objects that are acted upon by motor systems (in this case, eye movements) when selection is guided by object features. We now show an impairment of this in our patient ER.

Even less is known of the role of the thalamus in search, yet here we report a patient (AF) with extinction, with a general deficit in generating contralesional saccades in easy as well as more complex search displays. LaBerge [28] has implicated the thalamus as an important component of attentional circuits and LaBerge and Buchsbaum [29] showed elevated thalamic activity when subjects detected a letter. Ungerleider and Christensen [44] showed that pulvinar lesions in the monkey produced impairments in attentional scanning and Robinson et al. [42] argue that the dorsomedial nucleus of the pulvinar (where modulation is spatially selective) plays a role in selective attention. Finally, Perryman et al. [37] recorded from the pulvinar of the squirrel monkey and argued for the importance of this area for the convergence and integration of visual and oculomotor input and also its role in the mediation of visual attention. Indeed, it could be speculated that the anatomy of the pulvinar is perfect for this kind of attentive support since it projects retinotopically to all the major visual areas and also insofar as, like the parietal cortex, its different sub-divisions cover the modalities as though it might be interested in contributing to integration. Damage to this area may, therefore, lead to the symptoms described in AFs case.

We also had some interesting effects that occurred for all patients: saccade amplitudes towards the single target stimu- lus proved of normal length despite the fact that amplitudes towards targets on the left were generally shorter than those towards targets on the right. This could indicate that saccade execution and oculomotor control as such were not impaired, an observation in line with other studies [25]. However, the fact that all RCVA patients, apart from $\mathrm{MH}$, executed more saccades compared to controls could also mean that CVA patients might have reduced peripheral vision which forces them to perform saccades in order to achieve letter discrimination. Healthy subjects might not have such a need. However, if there should be such a need than our data indicate that the patients can clearly do so as there seems to be no oculomotor abnormality per se. For the RCVA control patient $\mathrm{MH}$, this was not the case. This patient produced eye movements in only $40 \%$ of the trials, they were clearly hypometric in amplitude and his reaction times were also slowed for targets appearing on the left. It is likely that these impairments are the result of his right frontal damage. Schiller [43] implicated the importance of the frontal eye field (FEF) for saccade generation and Lynch and McLaren [33] found that not only latencies of contralateral saccades were slowed after FEF lesions, but that the monkey also refrained from executing saccades into the contralateral field when presented with a single target. However, although this patient produced far fewer saccades which were hypometric, it has to be borne in mind that his saccadic performance was quite different from that of the two neglect patients in that for the saccades he did execute not left/right asymmetry became apparent when the percentage of correct saccades to target was analysed.

To conclude, the results of the present study demonstrate that while the reaction times of the two neglect patients and indeed the control patient $\mathrm{MH}$, showed similar patterns in that all patients responded more slowly to targets on the left-hand side of the search display, their saccadic search behaviour was clearly different. AF has a general deficit in generating contralesional saccades in search; this deficit appears to be little affected by the nature of the distractors. In contrast ERs difficulty in generating such saccades does appear to be distractor dependent. MH is hypokinetic, but shows no left/right asymmetry once a target directed saccade is executed. In the current study, the measurement of saccades, thus, allowed the task to be fractionated and reveal the action of multiple mechanism controlling saccades in search.

\section{Acknowledgements}

We would like to thank ER and AF and the other participants for their co-operation, Vince Walsh and Dave Perrett for advice on the neurophysiological sections and Robin Walker for most helpful comments on an earlier version of this manuscript. This research was supported by a Wellcome Trust grant awarded to M. Harvey (no. 050184/Z). B. Olk was solely funded by the Wellcome Trust (no. 050184/Z). 


\section{References}

[1] Aglioti S, Smania N, Barbieri C, Corbetta M. Influence of stimulus salience and attentional demands on visual search patterns in hemispatial neglect. Brain and Cognition 1997;34:388-403.

[2] Ashbridge E, Walsh V, Cowey A. Temporal aspects of visual search studied by transcranial magnetic stimulation. Neuropsychologia 1997;35:1121-31

[3] Behrmann M, Watt S, Black SE, Barton JJS. Impaired visual search in patients with unilateral neglect: an oculographic analysis. Neuropsychologia 1997;35:1445-58.

[4] Chedru F, Leblanc M, Lhermitte F. Visual searching in normal and brain-damaged subjects: contribution to the study of unilateral inattention. Cortex 1973;9:94-111.

[5] Chelazzi L, Miller EK, Duncan J, Desimone R. A neural basis for visual search in inferior temporal cortex. Nature 1993;363:345-7.

[6] Damasio H, Damasio AR. Lesion analysis in neuropsychology. Oxford: Oxford University Press, 1989.

[7] Desimone R, Chelazzi L, Miller EK, Duncan J. Neuronal mechanisms of visual attention. In: Papathomas TV, Chubb C, Gorea A, Kowler E, editors. Early vision and beyond. London: MIT Press, 1995. p. $219-26$

[8] Deubel H, Schneider WX. Saccade target selection and object recognition: evidence for a common attentional mechanism. Vision Research 1996;36:1827-37.

[9] Eglin M, Robertson LC, Knight RT. Visual search performance in the neglect syndrome. Journal of Cognitive Neuroscience 1989;1:372-85.

[10] Eglin M, Robertson LC, Knight RT, Brugger P. Search deficits in neglect patients are dependent on size of the visual scene. Neuropsychology 1994;8:451-63.

[11] Findlay JM. Saccade target selection during visual search. Vision Research 1997;37:617-31.

[12] Findlay JM, Walker R. Visual attention and saccadic eye movements in normal human subjects and in patients with unilateral neglect. In: Zangenmeister WH, Stiehl, HS, Freska C, editors. Visual attention and cognition. Amsterdam: Elsevier, 1996. p. 95-113.

[13] Findlay JM, Walker R. A model of saccade generation based on parallel processing and competitive inhibition. Behavioral and Brain Sciences 1999;22:661-721.

[14] Gaymard B, Ploner CJ, Rivaud S, Vermersch AI, Pierrot-Deseilligny C. Cortical control of saccades. Experimental Brain Research 1998;123:159-63.

[15] Gainotti G. The role of spontaneous eye movements in orienting of attention and in unilateral neglect. In: Robertson IH, Marshall JC, editors. Unilateral neglect: clinical and experimental studies. Hillsdale, NJ: Lawrence Erlbaum Associates, 1993. p. 107-22.

[16] Girotti F, Casazza M, Musicco M, Avanzini G. Oculomotor disorders in cortical lesions in man: the role of unilateral neglect. Neuropsychologia 1983;21:543-53.

[17] Grabowecky M, Robertson LC, Treisman A. Pre-attentive processes guide visual search: evidence from patients with unilateral visual neglect. Journal of Cognitive Neuroscience 1993;5:288-302.

[18] Heide W, Kömpf D. Combined deficits of saccades and visuo-spatial orientation after cortical lesions. Experimental Brain Research 1998;123:164-71.

[19] Hoffman JE, Subramaniam B. The role of visual attention in eye movements. Perception and Psychophysics 1995;57:787-95.

[20] Hornak J. Ocular exploration in the dark by patients with visual neglect. Neuropsychologia 1992;30:547-52.

[21] Ishiai $S$, Furukawa $T$, Tsukagoshi $H$. Eye-fixation patterns in homonumous hemianopia and unilateral spatial neglect. Neuropsychologia 1987;25:675-9.

[22] Jahnke MT, Denzler P, Liebelt B, Reichert H, Mauritz KH. Eye movements and fixation characteristics in perception of stationary scenes: normal subjects as compared with patients with visual neglect or hemianopia. European Journal of Neurology 1995;2:275-95.
[23] Karnath H-O. Blickbewegungsmuster während der visuellen Exploration einfacher Zeichnungen bei einem Patienten mit visuellem Neglect. Zeitschrift fuer Neuropsychologie 1993;4:11324.

[24] Karnath H-O, Fetter M. Ocular space exploration in the dark and its relationship to subjective and objective body orientation in neglect patients with parietal lesions. Neuropsychologia 1995;33:3717.

[25] Karnath HO, Niemeier M, Dichgans J. Space exploration in neglect. Brain 1998;121:2357-67.

[26] Kowler E, Anderson E, Dosher B, Blaser E. The role of attention in the programming of saccades. Vision Research 1995;35:1897-916.

[27] Kustov AA, Robinson DL. Shared neural control of attentional shifts and eye movements. Nature 1996;384:74-7.

[28] LaBerge D. Attentional processing. Nancy: University Press, 1995.

[29] LaBerge D, Buchsbaum MS. Positron emission tomographic measurements of pulvinar activity during an attention task. Journal of Neuroscience 1990;10:613-9.

[30] Làdavas E, Petronio A, Umilta C. The development of visual attention in the intact field of hemineglect patients. Cortex 1990;26:307-17.

[31] Làdavas E, Zeloni G, Zaccara G, Gangemi P. Eye movements and orienting of attention in patients with visual neglect. Journal of Cognitive Neuroscience 1997;9:67-74.

[32] Liversedge SP, Findlay JM. Saccadic eye movements and cognition. Trends in Cognitive Science 2000;4:6-14.

[33] Lynch JC, McLaren JW. Deficits of visual-attention and saccadic eye movements after lesions of parietooccipital cortex in monkeys Journal of Neurophysiology 1989;61:74-90.

[34] Olk B, Gilchrist ID, Harvey M. Influence of lower and higher-level factors on saccadic eye movements during visual search. Experimentelle Psychologie 2000;SS:149.

[35] Olk B, Harvey M, Dow L, Murphy PJS. Illusion processing in hemispatial neglect. Neuropsychologia 2001;39:611-25.

[36] Olk B, Harvey M. Effects of visible and invisible cueing on line bisection and landmark performance in hemispatial neglect. Neuropsychologia, in press.

[37] Perryman KM, Lindsley DF, Lindsley DB. Pulvinar neuron responses to spontaneous and trained eye movements and to light flashes in squirrel monkeys. Electroencephalogy and Clinical Neurophysiology 1980;49:152-61.

[38] Pierrot-Deseilligny C, Rivaud S, Gaymard B, Mueri R, Vermersch AI. Cortical control of saccades. Annals of Neurology 1995;37:55767.

[39] Remington RW. Attention and saccadic eye movements. Journal of Experimental Psychology: Human Perception and Performance 1980;6:726-44.

[40] Riddoch MJ, Humphreys GW. Perceptual and action systems in unilateral visual neglect. In: Jeannerod M, editor. Neurophysiological and neuropsychological aspects of spatial neglect. Amsterdam: Elsevier, 1987. p. 151-81.

[41] Rizzo M, Hurtig R. Visual search in hemineglect: what stirs idle eyes? Clinical Vision Sciences 1992;7:39-52.

[42] Robinson DL, Petersen SE, Keys W. Saccade related and visual activities in the pulvinar nuclei of the behaving rhesus monkey. Experimental Brain Research 1986;62:625-34.

[43] Schiller PH. The neural control of visually guided eye movements. In: Richards J, editor. Cognitive neuroscience, 1998.

[44] Ungerleider LG, Christensen CA. Pulvinar lesions in monkeys produce abnormal sanning of a complex visual array. Neuropsychologia 1979;17:493-501.

[45] Walker R, Young AW. Object-based neglect: an investigation of the contributions of eye movements and perceptual completion. Cortex 1996;32:279-95.

[46] Walker R, Findlay JM, Young AW, Lincoln NB. Saccadic eye movements in object-based neglect. Cognitive Neuropsychology 1996;13:569-615. 
[47] Williams DE, Reingold EM, Moscovitch M, Behrmann M. Patterns of eye movements during parallel and serial visual search tasks. Canadian Journal of Experimental Psychology 1997;51:151-64.

[48] Wilson B, Cockburn J, Halligan PW. Behavioural inattention test. Titchfield, Hampshire: Thames Valley Test Company, 1987.
[49] Wolfe JM. Guided search 2.0-a revised model of visual-search. Psychonomics Bulletin Review 1994;1:202-38.

[50] Zelinsky GJ, Sheinberg DL. Eye movements during parallel-serial visual search. Journal of Experimental Psychology: Human Perception and Performance 1997;23:244-62. 